\title{
Growth, Yield and Yield Attributes as Influenced by Treated Distillery Effluent Application in Maize (Zea mays L.) under Vertisols
}

\author{
D. Dinesh ${ }^{1 *}$, Dinesh Jinger ${ }^{1}$, K. Rajan², M. Sankar ${ }^{3}$, \\ R. Murugaragavan ${ }^{4}$ and Ram Partap ${ }^{1}$ \\ ${ }^{1}$ ICAR-Indian Institute of Soil and Water Conservation, RC, Vasad, Anand, Gujarat, India \\ ${ }^{2}$ ICAR-Indian Institute of Soil and Water Conservation, \\ $R C$, Udhagamandalam, TamilNadu, India \\ ${ }^{3}$ ICAR- Indian Institute of Soil and Water Conservation, Dehradun, Uttarakhand, India \\ ${ }^{4}$ Agriculture College and Research Institute, TNAU, Madurai, Tamil Nadu, India \\ *Corresponding author
}

\section{A B S T R A C T}

Keywords

Maize,

Zea mays,

Distillery Effluent

\section{Article Info}

Accepted:

12 December 2020

Available Online:

10 January 2021
A field experiment was conducted to find out the effect of treated distillery effluent (TDE) and bio-compost (BC) application on productivity of maize crop grown in vertisols having clay loam texture. Five levels each of organic fertilizers in main plot (control, TDE at 50 $\mathrm{kL} \mathrm{ha}^{-1}$, TDE at $100 \mathrm{~kL} \mathrm{ha}^{-1}, \mathrm{BC}$ at $5 \mathrm{t} \mathrm{ha}^{-1}$ and FYM at $12.5 \mathrm{t} \mathrm{ha}^{-1}+$ bio-fertilizers and inorganic fertilizers in sub plot (control, 50, 75, 100\% recommended dose (RD) of $\mathrm{N}$ (nitrogen) \& $\mathrm{P}$ (phosphorus) and $100 \% \mathrm{RD}$ of $\mathrm{N}+\mathrm{P}+\mathrm{K}$ (potassium) were tested in split plot design and replicated thrice. Application of TDE at $100 \mathrm{~kL} \mathrm{ha}^{-1}$ along with either $100 \% \mathrm{RD}$ of NPK or NP increased the grain and stover yield by $18.3-26.3$ and $37.6-47.15 \%$ respectively, over control. However, this treatment was found comparable with FYM at $12.5 \mathrm{t} \mathrm{ha}^{-1}+$ bio-fertilizers along with either $100 \%$ RD of NPK or NP. Similarly, dry matter production was also found maximum with the application of TDE at $100 \mathrm{~kL} \mathrm{ha}^{-1}$ and FYM $12.5 \mathrm{t} \mathrm{ha}^{-1}+$ bio-fertilizers. The growth and yield attributes of maize viz., plant height, leaf area index, cob length, cob girth, number of grain rows $\mathrm{cob}^{-1}$, number of grains row $^{-1}$, number of grains $\mathrm{cob}^{-1}, 100$-grains weight and cob weight were significantly increased by TDE application and the highest values were recorded at $100 \mathrm{~kL} \mathrm{ha}^{-1}$ along with $100 \%$ RD of NPK as well as NP fertilizers. TDE contain considerable level of plant nutrients which were made available to the plant, thus resulted in better growth and productivity yield of maize.

\section{Introduction}

Maize has its significance as a source of a large number of industrial products besides its uses as human food and animal feed. In India, maize is cultivated on 9.07 million hectares (Mh) with a production of 27.30 million tons (Mt) and the average yield is $2627 \mathrm{~kg} \mathrm{ha}^{-1}$
(Agricoop, 2018). By 2020, the requirement of maize for various sectors will be around $100 \mathrm{Mt}$, of which poultry sector needs $31 \mathrm{Mt}$. Therefore, to meet the demand to various sectors it is indispensable to increase the production of maize. Further, for increasing the production and productivity of crops synthetic fertilizers play a vital role. 
However, sole application of chemical fertilizer results in leaching and runoff of nutrients, especially $\mathrm{N}$ and $\mathrm{P}$ which leads to environmental degradation (Dass et al., 2014), adverse effect on soil properties and produce quality (Gupta et al., 2018). Therefore, integrated application of organic and inorganic sources of nutrients along with bio fertilizers on the other hand could provide a good substrate for crop growth and development through congenial nutrient supply environment to the crops (Choudhary et al., 2010).

In this regard, sugar and distillery complex generates large quantities of solid and liquid bio-wastes like bagasse (the fibre residue of sugarcane), press mud (filter cake), molasses and distillery effluent or spent wash. However, some of these wastes are recycled for agricultural purpose, mainly for reclamation of salt affected soil as well as for nutrient supplement to the various crops. Presently the distillery industrial wastes, which are considered unusable, have found to be useful in support of sustainable agriculture. At present, there are 319 distilleries in India with an installed capacity of 3.25 billion liters of alcohol and generating 48 billion litres of distillery effluent annually (Uppal, 2004; Dinesh et al., 2020).

In general, the treated effluent contains considerable amount of primary $(\mathrm{N} P, K)$, secondary $(\mathrm{Ca}, \mathrm{Mg}, \mathrm{S})$ and micro nutrients (Haroon and Bose, 2004). The distillery effluent after treatment $(\mathrm{pH}$ correction and BOD reduction) can be a good source of nutrients. The TDE improves the soil biological and biochemical activities (Murugaragavan, 2002). It has been also observed that application of distillery effluent in acid soil is not accounting for any change that affect the soil quality (Das et al., 2010). Therefore, this could be used as fertilizer for improving crop growth and soil fertility
(Suganya et al., 2002; Joshi et al., 2000). In order to harness its full manurial potential, there is a recommendation of one time land application of distillery effluent as a source of plant nutrients (Baskar et al., 2003). Keeping the above facts in view, the current study was aimed to find out the repose of TDE and BC application on maize grown under vertisols and their effect on growth yield and yield attributes.

\section{Materials and Methods}

\section{Experimental site and growing condition}

Field experiment was conducted in Tenkizhani village of Vellore district, Tamil Nadu. The soil of the study area belongs to Idayapatti series which is fine montmorillonitic hyperthermic family of Typic Haplusterts, vertisols. The geographical location of the experiment site was $12^{\circ} 51^{\prime} \mathrm{N}$ latitude $79^{\circ} 18^{\prime} \mathrm{E}$ longitudes with an altitude of $246 \mathrm{~m}$ above the mean sea level. In initial stage the soil was slightly alkaline ( $\mathrm{pH} \mathrm{8.4)}$ and EC of $0.30 \mathrm{dS} \mathrm{m}^{-1}$. The organic carbon content was found to be low $\left(7.40 \mathrm{~g} \mathrm{~kg}^{-1}\right)$. The $\mathrm{N}$ was low $\left(210 \mathrm{~kg} \mathrm{ha}^{-1}\right)$ and the $\mathrm{K}\left(309 \mathrm{~kg} \mathrm{ha}^{-}\right.$ $\left.{ }^{1}\right)$ and $\mathrm{P}\left(23 \mathrm{~kg} \mathrm{ha}^{-1}\right)$ level were high in status. The Mn (10.5 mg kg $\left.{ }^{-1}\right), \mathrm{Fe}\left(8.68 \mathrm{mg} \mathrm{kg}^{-1}\right), \mathrm{Cu}$ (3.00 mg kg-1) and hot water soluble Boron $\left(0.52 \mathrm{mg} \mathrm{kg}^{-1}\right)$ were found to be sufficient in the soil but the availability of $\mathrm{Zn}(0.67 \mathrm{mg} \mathrm{kg}-$ $\left.{ }^{1}\right)$ was found to be deficient. The microbial populations of experiment soils were $1.50 \times$ $10^{6} \mathrm{CFU} \mathrm{g}^{-1}$ of soil for bacteria, $8.20 \times 10^{4}$ $\mathrm{CFU} \mathrm{g}{ }^{-1}$ of soil for fungi and $4.5 \times 10^{2} \mathrm{CFU} \mathrm{g}^{-}$ ${ }^{1}$ of soil for Actinomycetes.

\section{Experimental design and treatments}

Five levels each of organic fertilizers in main plot (control, TDE at $50 \mathrm{~kL} \mathrm{ha}^{-1}$, TDE at 100 $\mathrm{kL} \mathrm{ha}^{-1}, \mathrm{BC}$ at $5 \mathrm{t} \mathrm{ha}^{-1}$ and FYM at $12.5 \mathrm{t} \mathrm{ha}^{-1}$ + bio-fertilizers and inorganic fertilizers in sub plot (control, 50, 75, 100\% RD of $\mathrm{N}+\mathrm{P}$ 
and $100 \% \mathrm{RD}$ of $\mathrm{N}+\mathrm{P}+\mathrm{K}$ were tested in split plot design and replicated thrice. The soil was ploughed well and the calculated quantities of TDE, BC and FYM as per the treatment schedule were applied uniformly to the concerned plots before 30 days of sowing. After 30 days, surface of the treated plots were inverted manually with spade to facilitate aeration and oxidation. Then, the ridges and furrows were formed as the test crop was maize (local farmers preferred variety used) and the fertilizer schedule of 150:75:75 kg N, $\mathrm{P}_{2} \mathrm{O}_{5}, \mathrm{~K}_{2} \mathrm{O}$ ha $^{-1}$ was adopted as $100 \%$ recommended dose (TNAU, 2007). Bio compost was prepared by utilizing the press mud (an organic solid material obtained as by-product from sugar industries) as a raw material and the composting process is being carried out by mechanized open window system with the use of treated distillery spent wash and bio inoculants for about 70-80 days. The product is then sun dried ground and sieved by mechanical separator and then used as BC for manuring purpose. Biofertilizers (Azospirillum and Phosphobacteria were used at $2.0 \mathrm{~kg} \mathrm{ha}^{-1}$ in respective treatment. Calculated quantities of fertilizers were applied in each treatment as per the treatment schedule. Entire dose of $\mathrm{P}$ was applied as basal while $\mathrm{N}$ and $\mathrm{K}$ were applied in two splits at 25 and 50 days after sowing (DAS). To avoid micro nutrients deficiency, the TNAU micronutrient mixture at $12.5 \mathrm{~kg}$ $\mathrm{ha}^{-1}$ as enriched with FYM was applied, uniformly. All other cultural practices including gap filling, thinning, weeding and plant protection measures were carried out as per the recommendations of crop production guide of Tamil Nadu Agricultural University.

\section{Biometric observations}

Plant samples were collected on 30 and 60 DAS and also at harvest stage. Five samples were collected from each treatment plot, chopped to small pieces separately, air dried for one week and then oven dried at $60-70^{\circ} \mathrm{C}$ till a constant weight was reached. The oven dry weight of each sample was determined and the total dry matter production (DMP) $\mathrm{kg}$ $\mathrm{ha}^{-1}$ was calculated. These samples were finally powdered and used for chemical analysis. Height of the plants were measured from the base of the plant to the tip of the top most opened leaf in ten representative plants at various stages of crop growth and expressed in $\mathrm{cm}$. From ten randomly selected plants in each treatment plot, maximum length and breadth of the fully opened third leaf from the top of the plant was measured. The total number of leaves in all the five plants was counted. The mean values were worked out for the maximum length, breadth and number of leaves plant ${ }^{-1}$. The leaf area index (LAI) was calculated by using the following formula as suggested by Mckee (1964).

$$
\mathrm{LAI}=\frac{\mathrm{L} \times \mathrm{B} \times \mathrm{N} \times 0.796}{\text { Spacing }(\mathrm{cm})}
$$

$\mathrm{L}$ - Length of the leaf $(\mathrm{cm})$

$\mathrm{N}$ - No. of leaves

B - Breath of the leaf $(\mathrm{cm})$

0.796 - Constant

The cobs from the net plot were harvested separately. The cobs were sun dried shelled, cleaned and grain yield were recorded for individual treatments and expressed in $\mathrm{kg}$ ha ${ }^{1}$. After the harvest of cobs, the stover in the net plot area were cut close to the ground level and left in the field for one week for sun drying. After drying, weight of stover from each treatment plot was recorded and expressed in $\mathrm{kg} \mathrm{ha}^{-1}$. Cob length, cob girth, grain rows $\mathrm{cob}^{-1}$, number of grains row ${ }^{-1}$, test weight ten representative plants at harvest were calculated according to standard procedure. The data of the experiment were statistically analyzed by adopting appropriate statistical methods as outlined by Gomez and Gomez (1984). The critical differences were 
calculated at $5 \%$ level of probability.

\section{Results and Discussion}

\section{Grain and stover yield}

Application of TDE, BC and FYM significantly affected the grain (Fig.1) and stover yield (Fig. 2). The results revealed that among the main plot treatments, application of TDE at $100 \mathrm{~kL} \mathrm{ha}^{-1}$ registered the highest grain $\left(5128 \mathrm{~kg} \mathrm{ha}^{-1}\right)$ and stover yield $(9813 \mathrm{~kg}$ $\left.\mathrm{ha}^{-1}\right)$. However, this treatment was comparable with FYM at $12.5 \mathrm{t} \mathrm{ha}^{-1}+$ biofertilizers. With respect to the organic manures viz., FYM and BC both of them were found comparable, while FYM was better than $\mathrm{BC}$ and both of them registered higher grain and stover yield than control. Causes of increase in yield under organic treatments were ascribed to synergism between biofertilizers and FYM, which increased the concentrations of $\mathrm{N}, \mathrm{P}$ and $\mathrm{K}$ ions in soil solution, which led to vigorous root development, and better growth and development of plant (Rathod et al., 2015). Among the subplot treatments application of $100 \%$ RD of NPK recorded the higher grain $\left(5510 \mathrm{ha}^{-1}\right)$ and stover yield $\left(10518 \mathrm{~kg} \mathrm{ha}^{-1}\right)$ compared to other treatments. However, this treatment was comparable with $100 \%$ RD of NP. The interaction of treatments was significant wherein the superiority of the application of TDE at $100 \mathrm{~kL} \mathrm{ha}^{-1}$ over the rest of the main plot treatments.

The effects of organic manure viz., BC and FYM were found comparable at all sub plot treatments as well as all the main plot treatments other than control. Hati et al., (2007) reported an increase in the grain yield of wheat in the distillery effluent applied treatments.

The distillery effluent is essentially of plant origin and contains high level of plant nutrients which were made available to the plant, thus resulting in better growth, development and yield of the crops. Similar effects with the usage of distillery effluent were also reported by Ramana et al., (2002); Dinesh et al., (2017) in maize.In contrast, Baskar et al., (2001) reported that the preplant application of distillery spent wash markedly increased the cane yield of sugarcane. Bhalerao et al., (2005) reported that the pre-sowing application of distillery spent wash recorded similar impact on the yield of Dhaincha and sugarcane.

\section{Growth parameters}

The dry matter production (DMP), plant height, number of leaves, leaf length and LAI of maize were significantly influenced by the application of TDE, BC, FYM and RD of NPK and NP. DMP and plant height ranged from 0.57 to $18.4 \mathrm{t} \mathrm{ha}^{-1}$ (Table 1) and $71.0 \mathrm{~cm}$ $201 \mathrm{~cm}$ (Table 2) respectively. Application of TDE and organic manures significantly increased the DMP and plant height of maize crop at all stages of observation. Application of TDE at $100 \mathrm{~kL} \mathrm{ha}^{-1}$ registered significantly higher DMP and plant height which was followed by FYM at $12.5 \mathrm{tha}^{-1}+$ bio-fertilizer and were found superior to control at almost all the stages of crop growth. Combination of organic sources of nutrients with biofertilizers results in more uptake of nutrients as compared to sole use of organic ones (Patil and Padmani, 2007).

This may be due to the fact that the balanced and combined use of various plant nutrient sources results in optimum absorption, translocation and assimilation of those nutrients. This ultimately leads to increase in the dry-matter accumulation and other growth parameters (Gupta et al., 2020). Kayalvizhi et al., (2001) observed higher values of growth parameters and cane yield due to application of distillery spentwash at 1:30 dilutions. 
Table.1 Effect of TDE, BC and FYM application on dry matter production (DMP) yield ( $\mathrm{t}$ ha $^{-1}$ ) of maize crop

\begin{tabular}{|c|c|c|c|c|c|c|c|c|c|c|c|c|c|c|c|c|c|c|}
\hline \multirow{2}{*}{ Treatments } & \multicolumn{6}{|c|}{ Vegetative Stage } & \multicolumn{6}{|c|}{ Flowering Stage } & \multicolumn{6}{|c|}{ Harvesting Stage } \\
\hline & $\mathrm{S}_{1}$ & $\mathrm{~S}_{2}$ & $\mathrm{~S}_{3}$ & $\mathrm{~S}_{4}$ & $\mathrm{~S}_{5}$ & Mean & $\mathrm{S}_{1}$ & $\mathrm{~S}_{2}$ & $\mathrm{~S}_{3}$ & $\mathrm{~S}_{4}$ & $\mathrm{~S}_{5}$ & Mean & $\mathrm{S}_{1}$ & $\mathrm{~S}_{2}$ & $\mathrm{~S}_{3}$ & $\mathrm{~S}_{4}$ & $\mathrm{~S}_{5}$ & Mean \\
\hline $\mathbf{M}_{1}$ & 0.57 & 0.77 & 0.96 & 1.04 & 1.18 & 0.90 & 3.3 & 4.1 & 4.2 & 4.7 & 5.1 & 4.3 & 7.1 & 9.9 & 11.1 & 12.3 & 13.4 & 10.7 \\
\hline $\mathbf{M}_{2}$ & 0.71 & 0.97 & 1.33 & 1.55 & 1.61 & 1.23 & 5.2 & 6.1 & 7.8 & 8.0 & 8.2 & 7.0 & 10.8 & 13.0 & 14.9 & 16.0 & 16.5 & 14.2 \\
\hline $\mathbf{M}_{3}$ & 0.91 & 1.07 & 1.44 & 1.60 & 2.03 & 1.41 & 5.2 & 7.7 & 8.0 & 9.7 & 10.0 & 8.1 & 11.4 & 14.8 & 16.5 & 18.0 & 18.4 & 15.8 \\
\hline $\mathbf{M}_{4}$ & 0.74 & 1.17 & 1.39 & 1.52 & 1.77 & 1.32 & 5.1 & 5.8 & 6.2 & 7.0 & 7.2 & 6.2 & 11.0 & 13.5 & 15.2 & 16.5 & 17.1 & 14.7 \\
\hline $\mathbf{M}_{5}$ & 0.66 & 1.06 & 1.29 & 1.62 & 1.71 & 1.27 & 5.2 & 6.6 & 7.1 & 7.5 & 8.6 & 7.0 & 11.1 & 14.0 & 15.8 & 18.0 & 18.5 & 15.5 \\
\hline \multirow[t]{2}{*}{ Mean } & 0.72 & 1.01 & 1.28 & 1.46 & 1.66 & 1.23 & 4.8 & 6.1 & 6.7 & 7.4 & 7.8 & 6.5 & 10.3 & 13.0 & 14.7 & 16.2 & 16.8 & 14.2 \\
\hline & \multicolumn{3}{|c|}{ SEd } & \multicolumn{3}{|c|}{$\mathrm{CD}(0.05)$} & \multicolumn{3}{|c|}{ SEd } & \multicolumn{3}{|c|}{$\mathrm{CD}(0.05)$} & \multicolumn{3}{|c|}{ SEd } & \multicolumn{3}{|c|}{$\mathrm{CD}(0.05)$} \\
\hline $\mathbf{M}$ & \multicolumn{3}{|c|}{0.04} & \multicolumn{3}{|c|}{0.10} & \multicolumn{3}{|c|}{0.11} & \multicolumn{3}{|c|}{0.2} & \multicolumn{3}{|c|}{0.45} & \multicolumn{3}{|c|}{1.0} \\
\hline $\mathbf{S}$ & \multicolumn{3}{|c|}{0.07} & \multicolumn{3}{|c|}{0.14} & \multicolumn{3}{|c|}{0.24} & \multicolumn{3}{|c|}{0.5} & \multicolumn{3}{|c|}{0.28} & \multicolumn{3}{|c|}{0.6} \\
\hline$M \times S$ & \multicolumn{3}{|c|}{0.15} & \multicolumn{3}{|c|}{0.30} & \multicolumn{3}{|c|}{0.48} & \multicolumn{3}{|c|}{1.0} & \multicolumn{3}{|c|}{0.71} & \multicolumn{3}{|c|}{1.5} \\
\hline$S \times M$ & \multicolumn{3}{|c|}{0.16} & \multicolumn{3}{|c|}{0.32} & \multicolumn{3}{|c|}{0.53} & \multicolumn{3}{|c|}{1.1} & & 0.62 & & & 1.3 & \\
\hline
\end{tabular}

Table.2 Effect of TDE, BC and FYM application on plant height (cm) of maize crop

\begin{tabular}{|c|c|c|c|c|c|c|c|c|c|c|c|c|}
\hline \multirow[t]{2}{*}{ Treatments } & \multicolumn{6}{|c|}{ Vegetative stage } & \multicolumn{6}{|c|}{ Flowering stage } \\
\hline & $\mathrm{S}_{1}$ & $\mathrm{~S}_{2}$ & $\mathrm{~S}_{3}$ & $\mathrm{~S}_{4}$ & $\mathrm{~S}_{5}$ & Mean & $\mathrm{S}_{1}$ & $\mathrm{~S}_{2}$ & $\mathrm{~S}_{3}$ & $\mathrm{~S}_{4}$ & $\mathrm{~S}_{5}$ & Mean \\
\hline $\mathbf{M}_{1}$ & 71.0 & 78.0 & 83.6 & 79.2 & 89.8 & 80.3 & 173 & 187 & 189 & 183 & 190 & 185 \\
\hline $\mathbf{M}_{2}$ & 73.9 & 77.1 & 82.1 & 92.8 & 92.8 & 83.7 & 183 & 195 & 195 & 190 & 200 & 193 \\
\hline $\mathbf{M}_{3}$ & 70.6 & 74.6 & 87.7 & 90.3 & 98.7 & 84.4 & 192 & 194 & 181 & 196 & 201 & 193 \\
\hline $\mathbf{M}_{4}$ & 71.6 & 78.3 & 83.7 & 91.6 & 92.2 & 83.5 & 191 & 186 & 193 & 190 & 196 & 191 \\
\hline $\mathbf{M}_{5}$ & 73.7 & 76.1 & 95.3 & 94.2 & 101.1 & 88.1 & 192 & 196 & 194 & 204 & 199 & 197 \\
\hline \multirow[t]{2}{*}{ Mean } & 72.2 & 76.8 & 86.5 & 89.6 & 94.9 & 84.0 & 186 & 192 & 190 & 193 & 197 & 192 \\
\hline & \multicolumn{3}{|c|}{ SEd } & \multicolumn{3}{|c|}{$\mathrm{CD}(0.05)$} & \multicolumn{3}{|c|}{ SEd } & \multicolumn{3}{|c|}{$\mathrm{CD}(0.05)$} \\
\hline M & \multicolumn{3}{|c|}{2.82} & \multicolumn{3}{|c|}{6.5} & \multicolumn{3}{|c|}{3.24} & \multicolumn{3}{|c|}{8} \\
\hline $\mathbf{S}$ & \multicolumn{3}{|c|}{3.40} & \multicolumn{3}{|c|}{6.9} & \multicolumn{3}{|c|}{2.39} & \multicolumn{3}{|c|}{5} \\
\hline $\mathbf{M} \times \mathbf{S}$ & \multicolumn{3}{|c|}{7.36} & \multicolumn{3}{|c|}{ NS } & \multicolumn{3}{|c|}{5.77} & \multicolumn{3}{|c|}{ NS } \\
\hline $\mathbf{S} \times \mathbf{M}$ & \multicolumn{3}{|c|}{7.60} & \multicolumn{3}{|c|}{ NS } & \multicolumn{3}{|c|}{5.33} & \multicolumn{3}{|c|}{ NS } \\
\hline
\end{tabular}


Table.3 Effect of TDE, BC and FYM application on number of leaves plant ${ }^{-1}$ of maize crop

\begin{tabular}{|c|c|c|c|c|c|c|c|c|c|c|c|c|}
\hline \multirow[t]{2}{*}{ Treatments } & \multicolumn{6}{|c|}{ Vegetative stage } & \multicolumn{6}{|c|}{ Flowering stage } \\
\hline & $\mathrm{S}_{1}$ & $\mathrm{~S}_{2}$ & $\mathrm{~S}_{3}$ & $\mathrm{~S}_{4}$ & $\mathrm{~S}_{5}$ & Mean & $\mathrm{S}_{1}$ & $\mathrm{~S}_{2}$ & $\mathrm{~S}_{3}$ & $\mathrm{~S}_{4}$ & $\mathrm{~S}_{5}$ & Mean \\
\hline $\mathbf{M}_{1}$ & 8.2 & 9.1 & 9.5 & 9.1 & 9.4 & 9.0 & 12.4 & 13.5 & 13.5 & 13.9 & 13.6 & 13.4 \\
\hline $\mathbf{M}_{2}$ & 8.8 & 9.1 & 9.1 & 9.7 & 9.7 & 9.3 & 12.9 & 13.9 & 13.5 & 13.9 & 13.7 & 13.6 \\
\hline $\mathbf{M}_{3}$ & 8.7 & 8.8 & 9.4 & 9.6 & 9.9 & 9.3 & 13.9 & 13.3 & 13.7 & 13.5 & 13.7 & 13.7 \\
\hline $\mathbf{M}_{4}$ & 8.6 & 9.6 & 9.2 & 9.9 & 10.1 & 9.5 & 13.7 & 13.5 & 13.7 & 13.9 & 13.9 & 13.7 \\
\hline $\mathbf{M}_{5}$ & 8.7 & 8.9 & 9.2 & 10.0 & 10.3 & 9.4 & 13.9 & 13.9 & 13.7 & 14.5 & 14.5 & 14.1 \\
\hline \multirow[t]{2}{*}{ Mean } & 8.6 & 9.1 & 9.3 & 9.7 & 9.9 & 9.3 & 13.4 & 13.6 & 13.6 & 13.9 & 13.9 & 13.7 \\
\hline & \multicolumn{3}{|c|}{ SEd } & \multicolumn{3}{|c|}{$\mathrm{CD}(0.05)$} & \multicolumn{3}{|c|}{ SEd } & \multicolumn{3}{|c|}{$\mathrm{CD}(0.05)$} \\
\hline M & \multicolumn{3}{|c|}{0.19} & \multicolumn{3}{|c|}{ NS } & \multicolumn{3}{|c|}{0.19} & \multicolumn{3}{|c|}{0.5} \\
\hline $\mathbf{S}$ & \multicolumn{3}{|c|}{0.23} & \multicolumn{3}{|c|}{0.5} & \multicolumn{3}{|c|}{0.16} & \multicolumn{3}{|c|}{0.3} \\
\hline$M \times S$ & \multicolumn{3}{|c|}{0.50} & \multicolumn{3}{|c|}{ NS } & \multicolumn{3}{|c|}{0.37} & \multicolumn{3}{|c|}{ NS } \\
\hline$S \times M$ & \multicolumn{3}{|c|}{0.52} & \multicolumn{3}{|c|}{ NS } & \multicolumn{3}{|c|}{0.35} & \multicolumn{3}{|c|}{ NS } \\
\hline
\end{tabular}

Table.4 Effect of TDE, BC and FYM application on leaf length $(\mathrm{cm})$ of maize crop

\begin{tabular}{|c|c|c|c|c|c|c|c|c|c|c|c|c|}
\hline \multirow[t]{2}{*}{ Treatments } & \multicolumn{6}{|c|}{ Vegetative stage } & \multicolumn{6}{|c|}{ Flowering stage } \\
\hline & $\mathrm{S}_{1}$ & $\mathrm{~S}_{2}$ & $\mathrm{~S}_{3}$ & $\mathrm{~S}_{4}$ & $\mathrm{~S}_{5}$ & Mean & $\mathrm{S}_{1}$ & $\mathrm{~S}_{2}$ & $\mathrm{~S}_{3}$ & $\mathrm{~S}_{4}$ & $\mathrm{~S}_{5}$ & Mean \\
\hline $\mathbf{M}_{1}$ & 55.0 & 59.8 & 62.3 & 59.5 & 64.4 & 60.2 & 74.3 & 78.5 & 76.0 & 79.1 & 74.6 & 76.5 \\
\hline $\mathbf{M}_{2}$ & 56.2 & 60.9 & 61.8 & 66.1 & 69.1 & 62.8 & 77.6 & 81.5 & 80.1 & 76.5 & 82.7 & 79.7 \\
\hline $\mathbf{M}_{3}$ & 54.5 & 56.4 & 65.7 & 69.5 & 74.0 & 64.0 & 79.7 & 82.1 & 84.2 & 82.2 & 84.4 & 82.5 \\
\hline $\mathbf{M}_{4}$ & 54.9 & 63.5 & 61.6 & 69.1 & 73.6 & 64.5 & 79.1 & 80.2 & 80.5 & 78.8 & 83.0 & 80.3 \\
\hline $\mathbf{M}_{5}$ & 54.8 & 60.6 & 70.9 & 69.1 & 76.1 & 66.3 & 78.8 & 81.7 & 80.9 & 82.7 & 83.8 & 81.6 \\
\hline \multirow[t]{2}{*}{ Mean } & 55.1 & 60.2 & 64.5 & 66.6 & 71.4 & 63.6 & 77.9 & 80.8 & 80.3 & 79.8 & 81.7 & 80.1 \\
\hline & \multicolumn{3}{|c|}{ SEd } & \multicolumn{3}{|c|}{$\mathrm{CD}(0.05)$} & \multicolumn{3}{|c|}{ SEd } & \multicolumn{3}{|c|}{$\mathrm{CD}(0.05)$} \\
\hline M & \multicolumn{3}{|c|}{2.73} & \multicolumn{3}{|c|}{ NS } & \multicolumn{3}{|c|}{1.21} & \multicolumn{3}{|c|}{2.8} \\
\hline $\mathbf{S}$ & \multicolumn{3}{|c|}{5.46} & \multicolumn{3}{|c|}{5.0} & \multicolumn{3}{|c|}{1.84} & \multicolumn{3}{|c|}{ NS } \\
\hline $\mathbf{M} \times \mathbf{S}$ & \multicolumn{3}{|c|}{5.62} & \multicolumn{3}{|c|}{ NS } & \multicolumn{3}{|c|}{3.87} & \multicolumn{3}{|c|}{ NS } \\
\hline S $x$ M & \multicolumn{3}{|c|}{5.50} & \multicolumn{3}{|c|}{ NS } & \multicolumn{3}{|c|}{4.11} & \multicolumn{3}{|c|}{ NS } \\
\hline
\end{tabular}


Table.5 Effect of TDE, BC and FYM application on LAI of maize crop

\begin{tabular}{|c|c|c|c|c|c|c|c|c|c|c|c|c|}
\hline \multirow[t]{2}{*}{ Treatments } & \multicolumn{6}{|c|}{ Vegetative stage } & \multicolumn{6}{|c|}{ Flowering stage } \\
\hline & $\mathrm{S}_{1}$ & $\mathrm{~S}_{2}$ & $\mathrm{~S}_{3}$ & $\mathrm{~S}_{4}$ & $\mathrm{~S}_{5}$ & Mean & $\mathrm{S}_{1}$ & $\mathrm{~S}_{2}$ & $\mathrm{~S}_{3}$ & $\mathrm{~S}_{4}$ & $\mathrm{~S}_{5}$ & Mean \\
\hline $\mathbf{M}_{1}$ & 1.63 & 2.14 & 2.48 & 2.07 & 2.63 & 2.19 & 5.69 & 6.11 & 6.14 & 6.18 & 6.19 & 6.06 \\
\hline $\mathbf{M}_{2}$ & 1.94 & 2.26 & 2.21 & 3.12 & 3.18 & 2.54 & 6.09 & 7.08 & 7.10 & 7.18 & 7.21 & 6.93 \\
\hline $\mathbf{M}_{4}$ & 1.69 & 2.75 & 2.31 & 3.11 & 3.70 & 2.71 & 6.06 & 7.09 & 7.11 & 7.16 & 7.18 & 6.92 \\
\hline $\mathbf{M}_{5}$ & 1.83 & 2.09 & 3.14 & 3.30 & 3.81 & 2.84 & 6.05 & 7.20 & 7.24 & 7.31 & 7.38 & 7.04 \\
\hline Mean & 1.74 & 2.20 & 2.61 & 2.99 & 3.40 & 2.59 & 6.19 & 6.94 & 6.97 & 7.04 & 7.07 & 6.87 \\
\hline $\mathbf{M}$ & \multicolumn{3}{|c|}{0.26} & \multicolumn{3}{|c|}{ NS } & \multicolumn{3}{|c|}{0.21} & \multicolumn{3}{|c|}{0.49} \\
\hline $\mathbf{S}$ & \multicolumn{3}{|c|}{0.30} & \multicolumn{3}{|c|}{0.61} & \multicolumn{3}{|c|}{0.19} & \multicolumn{3}{|c|}{0.38} \\
\hline $\mathbf{M} \times \mathbf{S}$ & \multicolumn{3}{|c|}{0.66} & \multicolumn{3}{|c|}{ NS } & \multicolumn{3}{|c|}{0.43} & \multicolumn{3}{|c|}{ NS } \\
\hline $\mathbf{S} \times \mathbf{M}$ & \multicolumn{3}{|c|}{0.67} & \multicolumn{3}{|c|}{ NS } & \multicolumn{3}{|c|}{0.42} & \multicolumn{3}{|c|}{ NS } \\
\hline
\end{tabular}

Table.6 Effect of TDE, BC and FYM application on cob length and girth (cm) of maize crop

\begin{tabular}{|c|c|c|c|c|c|c|c|c|c|c|c|c|}
\hline \multirow[t]{2}{*}{ Treatments } & \multicolumn{6}{|c|}{ Cob length } & \multicolumn{6}{|c|}{ Cob girth } \\
\hline & $\mathrm{S}_{1}$ & $\mathrm{~S}_{2}$ & $\mathrm{~S}_{3}$ & $\mathrm{~S}_{4}$ & $\mathrm{~S}_{5}$ & Mean & $\mathrm{S}_{1}$ & $\mathrm{~S}_{2}$ & $\mathrm{~S}_{3}$ & $\mathrm{~S}_{4}$ & $\mathrm{~S}_{5}$ & Mean \\
\hline $\mathbf{M}_{1}$ & 16.7 & 17.4 & 17.0 & 16.8 & 18.0 & 17.2 & 13.0 & 13.4 & 13.0 & 13.2 & 13.7 & 13.2 \\
\hline $\mathbf{M}_{2}$ & 17.5 & 19.0 & 19.0 & 19.3 & 19.5 & 18.9 & 14.0 & 14.9 & 14.6 & 14.8 & 14.6 & 14.6 \\
\hline $\mathbf{M}_{3}$ & 18.1 & 19.4 & 18.8 & 19.0 & 19.5 & 19.0 & 14.0 & 14.9 & 14.8 & 14.5 & 14.7 & 14.6 \\
\hline $\mathbf{M}_{4}$ & 17.9 & 17.9 & 18.0 & 18.5 & 18.9 & 18.3 & 14.0 & 14.1 & 14.4 & 14.5 & 14.8 & 14.4 \\
\hline $\mathbf{M}_{5}$ & 17.5 & 19.5 & 19.4 & 20.2 & 20.4 & 19.4 & 13.4 & 14.2 & 14.2 & 14.5 & 14.2 & 14.1 \\
\hline \multirow[t]{2}{*}{ Mean } & 17.5 & 18.6 & 18.5 & 18.8 & 19.3 & 18.5 & 13.7 & 14.3 & 14.2 & 14.3 & 14.4 & 14.2 \\
\hline & \multicolumn{3}{|c|}{ SEd } & \multicolumn{3}{|c|}{$\mathrm{CD}(0.05)$} & \multicolumn{3}{|c|}{ SEd } & \multicolumn{3}{|c|}{$\mathrm{CD}(0.05)$} \\
\hline $\mathbf{M}$ & \multicolumn{3}{|c|}{0.31} & \multicolumn{3}{|c|}{0.7} & \multicolumn{3}{|c|}{0.33} & \multicolumn{3}{|c|}{0.8} \\
\hline $\mathbf{S}$ & \multicolumn{3}{|c|}{0.39} & \multicolumn{3}{|c|}{0.8} & \multicolumn{3}{|c|}{0.26} & \multicolumn{3}{|c|}{0.5} \\
\hline $\mathbf{M} \times \mathbf{S}$ & \multicolumn{3}{|c|}{0.84} & \multicolumn{3}{|c|}{1.7} & \multicolumn{3}{|c|}{0.62} & \multicolumn{3}{|c|}{1.3} \\
\hline $\mathbf{S} \times \mathbf{M}$ & \multicolumn{3}{|c|}{0.87} & \multicolumn{3}{|c|}{1.8} & \multicolumn{3}{|c|}{0.59} & \multicolumn{3}{|c|}{1.2} \\
\hline
\end{tabular}


Table.7 Effect of TDE, BC and FYM application on cob weight and 100-grain weight (g) of maize crop

\begin{tabular}{|c|c|c|c|c|c|c|c|c|c|c|c|c|}
\hline \multirow{2}{*}{ Treatments } & \multicolumn{6}{|c|}{ Cob Weight } & \multicolumn{6}{|c|}{100 grain weight } \\
\hline & $S_{1}$ & $\mathrm{~S}_{2}$ & $\mathrm{~S}_{3}$ & $\mathrm{~S}_{4}$ & $\mathrm{~S}_{5}$ & Mean & $\mathrm{S}_{1}$ & $\mathrm{~S}_{2}$ & $\mathrm{~S}_{3}$ & $\mathrm{~S}_{4}$ & $\mathrm{~S}_{5}$ & Mean \\
\hline $\mathbf{M}_{1}$ & 63 & 90 & 93 & 109 & 118 & 94 & 22.3 & 23.6 & 24.1 & 25.3 & 25.7 & 24.2 \\
\hline $\mathbf{M}_{2}$ & 73 & 95 & 104 & 107 & 128 & 101 & 24.5 & 25.7 & 27.3 & 27.3 & 27.7 & 26.5 \\
\hline $\mathbf{M}_{3}$ & 88 & 101 & 110 & 121 & 130 & 110 & 25.4 & 26.5 & 27.9 & 27.9 & 28.3 & 27.2 \\
\hline $\mathbf{M}_{4}$ & 72 & 79 & 87 & 113 & 125 & 95 & 23.0 & 23.0 & 26.9 & 26.5 & 27.6 & 25.4 \\
\hline $\mathbf{M}_{5}$ & 77 & 96 & 109 & 121 & 127 & 106 & 24.5 & 26.1 & 27.3 & 27.3 & 29.0 & 26.8 \\
\hline \multirow[t]{2}{*}{ Mean } & 75 & 92 & 101 & 114 & 126 & 101 & 23.9 & 25.0 & 26.7 & 26.9 & 27.6 & 26.0 \\
\hline & \multicolumn{3}{|c|}{ SEd } & \multicolumn{3}{|c|}{$\mathrm{CD}(0.05)$} & \multicolumn{3}{|c|}{ SEd } & \multicolumn{3}{|c|}{$\mathrm{CD}(0.05)$} \\
\hline M & \multicolumn{3}{|c|}{2.85} & \multicolumn{3}{|c|}{7} & \multicolumn{3}{|c|}{0.56} & \multicolumn{3}{|c|}{1.3} \\
\hline $\mathbf{S}$ & \multicolumn{3}{|c|}{6.01} & \multicolumn{3}{|c|}{12} & \multicolumn{3}{|c|}{0.36} & \multicolumn{3}{|c|}{0.7} \\
\hline$M \times S$ & \multicolumn{3}{|c|}{12.36} & \multicolumn{3}{|c|}{25} & \multicolumn{3}{|c|}{0.92} & \multicolumn{3}{|c|}{1.9} \\
\hline $\mathbf{S} \times \mathbf{M}$ & \multicolumn{3}{|c|}{13.44} & \multicolumn{3}{|c|}{27} & \multicolumn{3}{|c|}{0.81} & \multicolumn{3}{|c|}{1.6} \\
\hline
\end{tabular}

Table.8 Effect of TDE, BC and FYM application on number of grain row $\mathrm{cob}^{-1}$, number of grains per row ${ }^{-1}$ of maize crop

\begin{tabular}{|c|c|c|c|c|c|c|c|c|c|c|c|c|}
\hline \multirow[t]{2}{*}{ Treatments } & \multicolumn{6}{|c|}{ No. of grain row per cob ${ }^{-1}$} & \multicolumn{6}{|c|}{ No. of grains per row ${ }^{-1}$} \\
\hline & $\mathrm{S}_{1}$ & $\mathrm{~S}_{2}$ & $\mathrm{~S}_{3}$ & $\mathrm{~S}_{4}$ & $\mathrm{~S}_{5}$ & Mean & $\mathrm{S}_{1}$ & $\mathrm{~S}_{2}$ & $\mathrm{~S}_{3}$ & $\mathrm{~S}_{4}$ & $\mathrm{~S}_{5}$ & Mean \\
\hline $\mathbf{M}_{1}$ & 13.2 & 13.3 & 13.5 & 13.9 & 13.7 & 13.5 & 25.5 & 27.8 & 27.7 & 27.9 & 28.8 & 27.5 \\
\hline $\mathbf{M}_{2}$ & 14.1 & 14.1 & 14.6 & 14.3 & 14.3 & 14.3 & 28.1 & 30.3 & 31.4 & 30.8 & 32.4 & 30.6 \\
\hline $\mathbf{M}_{3}$ & 14.0 & 14.9 & 14.5 & 14.5 & 15.3 & 14.6 & 29.7 & 31.9 & 30.6 & 31.2 & 33.2 & 31.3 \\
\hline $\mathbf{M}_{4}$ & 14.4 & 14.6 & 13.8 & 14.2 & 14.1 & 14.2 & 28.1 & 30.8 & 30.8 & 29.4 & 31.6 & 30.1 \\
\hline $\mathbf{M}_{5}$ & 14.3 & 14.3 & 14.8 & 14.6 & 15.0 & 14.6 & 27.7 & 31.8 & 31.7 & 33.2 & 33.5 & 31.6 \\
\hline \multirow[t]{2}{*}{ Mean } & 14.0 & 14.2 & 14.2 & 14.3 & 14.5 & 14.2 & 27.8 & 30.5 & 30.4 & 30.5 & 31.9 & 30.2 \\
\hline & \multicolumn{3}{|c|}{ SEd } & \multicolumn{3}{|c|}{$\mathrm{CD}(0.05)$} & \multicolumn{3}{|c|}{ SEd } & \multicolumn{3}{|c|}{$\mathrm{CD}(0.05)$} \\
\hline M & \multicolumn{3}{|c|}{0.19} & \multicolumn{3}{|c|}{0.45} & \multicolumn{3}{|c|}{0.36} & \multicolumn{3}{|c|}{0.84} \\
\hline $\mathbf{S}$ & \multicolumn{3}{|c|}{0.20} & \multicolumn{3}{|c|}{0.40} & \multicolumn{3}{|c|}{0.47} & \multicolumn{3}{|c|}{0.96} \\
\hline$M \times S$ & \multicolumn{3}{|c|}{0.44} & \multicolumn{3}{|c|}{0.92} & \multicolumn{3}{|c|}{1.01} & \multicolumn{3}{|c|}{2.08} \\
\hline SxM & \multicolumn{3}{|c|}{0.44} & \multicolumn{3}{|c|}{0.90} & \multicolumn{3}{|c|}{1.06} & \multicolumn{3}{|c|}{2.14} \\
\hline
\end{tabular}



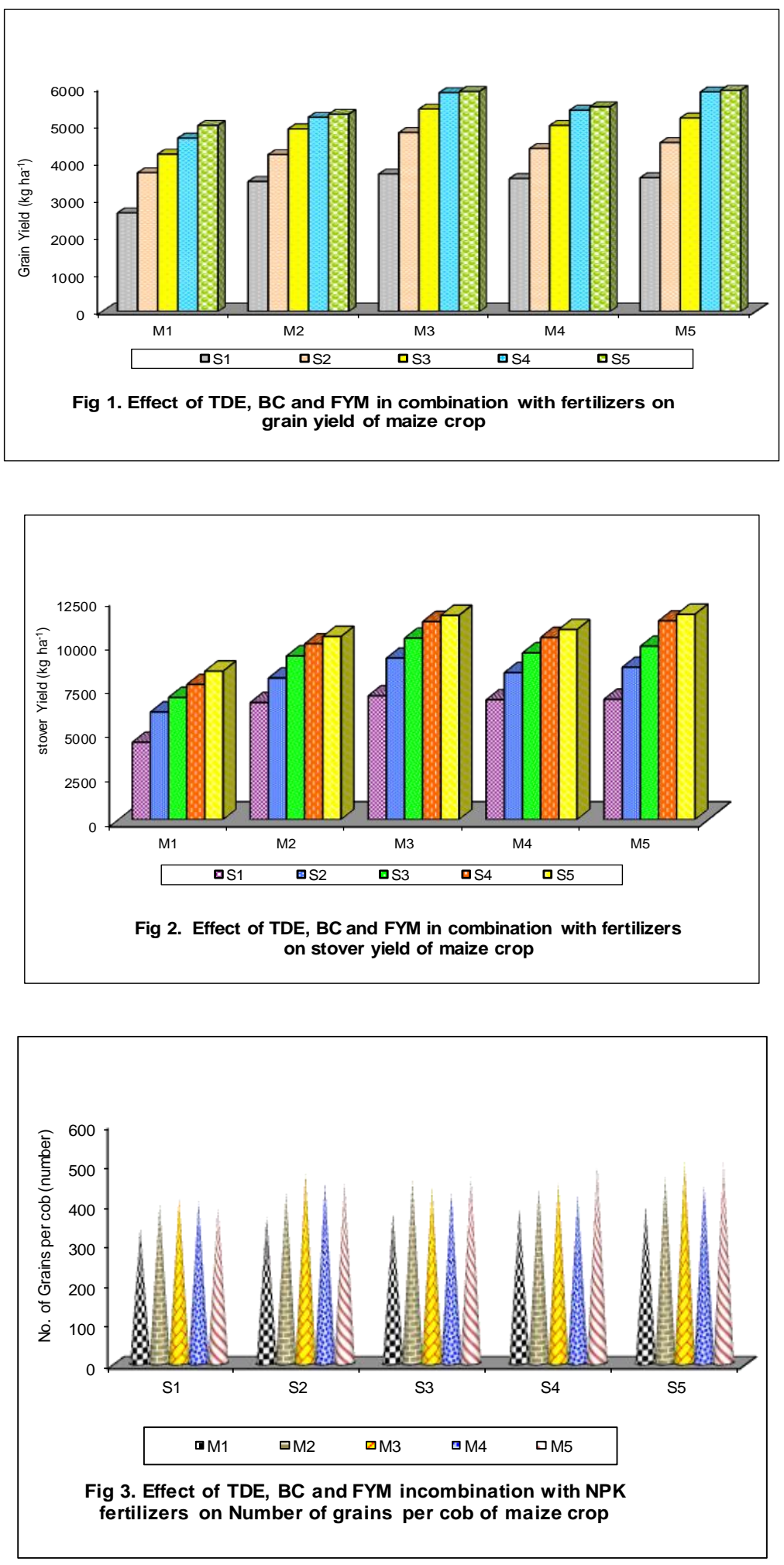
Dinesh et al., (2017) revealed that application of distillery effluent after tillering phase registered significantly higher plant height and DMP. With respect to the subplot treatments, application of $100 \%$ RD of NPK recorded highest DMP and plant height however it was comparable with $100 \%$ RD of NP in some extent and which were superior to all other subplot treatments at almost all the stages of observation. The interaction effect was significant at all the stages of observation. Application of TDE at $100 \mathrm{~kL}$ $\mathrm{ha}^{-1}$ along with $100 \%$ RD of NPK recorded a marked increase in the DMP and plant height at all the stage of observation followed by the application of TDE at $100 \mathrm{~kL} \mathrm{ha}^{-1}$ along with $100 \%$ RD of NP. Similarly, number of leaves, leaf length and LAI of maize crop followed the same pattern as followed under DMP and plant height. Number of leaves, leaf length and LAI ranged from 8.20 to 14.5 (Table 3), 55.0 to $84.4 \mathrm{~cm}$ (Table 4), 1.63-6.87 (Table 5) respectively. However, interaction effect was insignificant in these parameters. Ramana et al., (2002) reported that the application of distillery effluent significantly increased the DMP, plant height and other growth parameters of maize over control. The distillery spentwash is essentially a plant extract containing larger amount of most of the plant nutrients. Upon its application, the spentwash not only added plant nutrients, but also favoured mineralization of the nutrients in soil due to enhanced microbial activities. Increase in the availability of these nutrients might have facilitated greater uptake of nutrients by maize which reflected on better growth, development and yield of the crop.

\section{Yield attributes}

The yield attributes like cob length and cob girth were significantly influenced by the application of TDE and organic manures. The cob length and cob girth ranged from 16.7 to $19.5 \mathrm{~cm}$ and 13.0 to $14.7 \mathrm{~cm}$ respectively
(Table 6). The length and girth of cob was found to be higher in the plots which received TDE at $100 \mathrm{~kL} \mathrm{ha}^{-1}$ and found on par with FYM at $12.5 \mathrm{t} \mathrm{ha}^{-1}+$ bio-fertilizer. FYM applied at sufficient amount along with biofertilizers provides optimum favorable conditions for nutrient release as well as uptake and hence, due to the better response from plants it performed significantly (Pandey et al., 2015). The enhancement in yield attributing characters might be due to improved microbial activity in the rhizosphere with the application of organic manure and bio-fertilizer in conjunction which resulted in balanced nutrient supply, good microbial activity, and optimum moisture availability, anti-pathogenic activity resulting in better growth, yield attributes and yield (Reddy et al., 2011; Gupta et al., 2018). Among the subplot treatments, application of $100 \%$ RD of NPK recorded higher cob length and girth, however, found at par with $100 \%$ RD of NP. This was due to the increasing doses of chemical fertilizers contributing to the available nutrient pool in the soil which inurn increased the growth and yield attributes of the crop (Janaki, 2008). Cob weight and 100grain weight (Table 7) were also followed the same fashion as followed in case of cob length and cob girth. The interaction effect was also significant in the treatment combinations. Application of TDE at $100 \mathrm{~kL}$ $\mathrm{ha}^{-1}$ along with $100 \%$ RD of NPK followed by $100 \mathrm{~kL} \mathrm{ha}^{-1}$ along with $100 \%$ RD of NP found to be significant with themselves. The number of grains $\mathrm{cob}^{-1}$ (Fig. 3) number of grain rows $\mathrm{cob}^{-1}$ and number of grains row $^{-1}$ (Table 8) were also significantly influenced by application of TDE and organic manures. The number of grains $\mathrm{cob}^{-1}$, number of grain rows $\mathrm{cob}^{-1}$ and number of grains row ${ }^{-1}$ ranged from 336 to $506,13.2$ to 15.3 and 25.5 to 33.2 respectively. The application of TDE at $100 \mathrm{~kL} \mathrm{ha}^{-1}$ recorded the highest values of these attributes and found at par with FYM @ 12.5 $\mathrm{t} \mathrm{ha}^{-1}+$ bio-fertilizers. Among the 
subplot treatments, application of $100 \%$ RD of NPK recorded the highest values of these attributes and found at par with $100 \% \mathrm{RD}$ of NP. Chemical fertilizers are concentrated nutrient suppliers which supply plant nutrients in quick manner even with the lower doses (Sahayet al., 2016) and leading to higher photosynthetic activity and translocation of photosynthates to the sink which in turn resulted in optimum development of yield attributes and finally in higher grain and straw yield (Goud and Kale, 2010). The number of grain rows per cob was found to be significantly influenced by the application of TDE and organic manures. The interaction effect was also significant. Application of TDE at $100 \mathrm{~kL} \mathrm{ha}^{-1}$ along with $100 \% \mathrm{RD}$ of NPK resulted in higher values of these parameters followed by FYM at $12.5 \mathrm{t} \mathrm{ha}^{-1}+$ biofertilizers along with 100\% RD of NPK and these treatments were found superior to control.

In conclusion the application of TDE at 100 $\mathrm{kL} \mathrm{ha}^{-1}$ along with either $100 \%$ RD of NPK or NP recorded highest grain and stover yield. However, application of FYM at $12.5 \mathrm{t} \mathrm{ha}^{-1}$ along with either $100 \%$ RD of NPK or $100 \%$ NP found significantly at par because this treatment performed equally better as that of $100 \%$ NPK and it could be inferred that complete skipping of $\mathrm{K}$ fertilization is also possible if integrated nutrient management involving FYM and bio-fertilizers are practiced. These practices not only save the $\mathrm{K}$ fertilizer inputs but also could considerably cut short the fertilizer import bills.

\section{References}

Baskar, M., Gopal, H., Kayalvizhi,C., Dawood, M.S., Bose, S.C. and Rajukkannu, K. 2001. Influence of pre-plant application of distillery effluent and fertilizers on soil properties and yield of sugarcane. In:
National seminar on use of poor quality water and sugar industrial effluents in agriculture, ADAC \& RI, Tamil Nadu Agric. Univ., Tiruchirapalli, February 5, pp. 76-82.

Baskar, M., Kayalvizhi, C. and Bose, S.C. 2003.Eco-friendly utilisation of distillery effluent in Agriculture-a review. Agricultural Reviews24(1): $16-30$.

Bhalerao, V.P., Jadhav, M.B. and Bhoi, P.G. 2005. Effect of distillery's biomethanated effluent on yield, quality of adsali suagarcane and soil properties. Cooperative Sugar 36(8):653-659.

Das, M., Chakraborty, H., Singandhupe, R.B., Muduli, S.D. and Kumar, A. 2010. Utilization of distillery wastewater for improving production in unproductive paddy growing area of India. Journal of Scientific and Industrial Research 69: 560-63.

Dass, A., Suri, V.K. and Choudhary, A. K. 2014. Site-specific nutrient management approaches for enhanced nutrient-use efficiency in agricultural crops: Research and Reviews. Journal of Crop Science and Technology 3(3):1-6.

Dinesh, D. Chithra, L. Baskar, M. Rajan, K. Senthilraja, K. Sankar, M. Kumar, R. Sivakumar, K. 2017. Variation of soil microbial growth and enzyme activities by application of treated distillery effluent in maize crop grown under sandy loam soils. International Journalof Current Microbiology and Applied Sciences 6: 1334-1348.

Dinesh, D., Velu, V., Kakade, V., Jinger, D. and Murugaragavan, R. 2020. Response of treated distillery effluent application on growth and yield attributes of maize (Zea mays L.) under Typic Rhodustalfs of Tamil Nadu. Annals of Agricultural Research 
41(1): 29-36

Gomez, K.A. and Gomez, A.A. 1984. Statistical procedure for Agricultural Research. New York: John Willey and Sons Publications.

Goud, V.V. and Kale, H.B. 2010. Productivity and profitability of pigeonpea under different sources of nutrientsin rainfed condition of Central India. Journal of Food Legumes 23(3 \& 4):212-7.

Gupta, G., Dhar, S., Dass, A., Sharma, V.K., Singh, R.K., Kumar, A., Jinger, D. and Kumar, A. 2018. Influence of bioinoculant mediated organic nutrient management on productivity and profitability of pigeonpea (Cajanus cajan) in a semi-arid agro-ecology. Indian Journal of Agricultural Sciences. 88(10): 1593-6.

Gupta, G., Dhar, S., Dass, A., Sharma, V.K., Singh, Shukla, L., Singh, R., Kumar, A., Kumar, A., Jinger, D., Sannagoudar, M.S., Kamboj, N.K. and Verma, G. 2020. Assessment of bioinoculant mediated nutrient management in terms of productivity, profitability and nutrient harvest index of pigeonpea-wheat cropping system in India. Journal of Plant Nutrition 43(17): 1-18.

Haroon, A. and Bose, S.C. 2004. Use of distillery spent wash for alkali soil reclamation, treated distillery effluent for fertigation of crops. Indian farming 53(11): 48-51.

Hati, K.M., Biswas, A.K., Bandyopadhyay, K.K. and Misra, A.K. 2007.Soil properties and crop yields on a vertisol in India with application of distillery effluent.

Soil Tillage Research 92:60-68.

Janaki, D. 2008. Utilization of distillery Spent wash as a manure to crops and its impact on soil, crop and ground water quality. Ph.D. Thesis, Tamil Nadu Agricultural University, Coimbatore-
3.

Joshi, H.C, Pathak, H., Chaudhary, A., Joshi, T.P., Phogat, V.K. and Kalra, N. 2000. Changes in soil properties with distillery effluent irrigation, Journal of Environmental Research 6:153-162.

Kayalvizhi, C., Gopal, H., Baskar, M., Dawood, M.S., Bose, S.C. and Rajukkannu, K. 2001.Effect of fertigation with distillery effluent on soil properties and yield of sugarcane.In: Proceedings of national seminar on "Use of poor quality water and sugar industrial effluents in Agriculture", held at AnbilDharmalingam Agricultural College and Research Institute, Tiruchirappalli-9, Tamil Nadu Agricultural University, Tamil Nadu, February 5, pp.75.

McKee, G.W. 1964. A co-efficient for computing leaf area in hybrid maize. Agronomy

Journal 56: $240-244$.

Murugaragavan, R. 2002. Distillery spentwash on crop production in dryland soils. M.Sc. Thesis, Tamil Nadu Agricultural University, Coimbatore.

Pandey, I.B., Pandey, R.K. and Kumar, R. 2015. Integrated nutrient management for enhancing productivity and profitability of long-duration pigeonpea (Cajanus cajan) under rainfed condition. Indian Journal of Agronomy 60(3):436-42.

Patil, A.B., and Padmani, D.R. 2007. Effect of IPM practices on yield, quality and economics of pigeon pea(Cajanus cajan L.) under rainfed conditions. International Journal of Agriculture Sciences 3(2):202-4.

Ramana, S., Biswas, A. K., Kundu, S., Sana, J.K. and Yadava, R.B.R. 2002. Effect of distillery effluent on seed germination in some vegetable crops. 
Bioresource Technology82(3): 273275.

Rathod, P.S., Sharma, A., Patil, D.H. and Dodamani, B.M. 2015. Performance of pigeonpea under different sourcesof nutrients in rainfed conditions of Karnataka. Journal of Food Legumes 28(2):43-5.

Reddy, A.S.R., Reddy, M.C.S., Babu, J.S., Khan, M.M. and Rao.M.M. 2011. Integrated nutrient management in pigeonpea (Cajanus cajan). International Journal of Applied Biology and Pharmaceutical Technology 2(2):467-70.

Sahay, A., Pratap, T., Tyagi, S., Nanher, A.H., Singh, R., Singh, S.S. and Singh, V.D. 2016. Effect of integrated nutrient management on growth, yield and quality of pigeonpea (Cajanus cajan L.) c.v. PUSA 9. The Bioscan: An International Quarterly Journal of Life Sciences 11(1):293-6.

Suganya, T.S., Meli, S.S. and Patil, R.H. 2002. Effect of spent wash as irrigation water on performance of maize (Zea mays) and soil fertility, Indian Journal of Agricultural Sciences 72: 73-78.

TNAU, 2007.Crop production guide. Tamil Nadu agricultural University, TNAU Press, Coimbatore-3.

Uppal, J. 2004.Waste utilization and effluent treatment in the Indian alcohol industry-an overview. In: Proceedings of Indo-EU workshop on promoting efficient water use in agro-based industries, New Delhi, Jan, 15-16. pp. $12-15$.

\section{How to cite this article:}

Dinesh, D., Dinesh Jinger, K. Rajan, M. Sankar, R. Murugaragavan and Ram Partap. 2021. Growth, Yield and Yield Attributes as Influenced by Treated Distillery Effluent Application in Maize (Zea mays L.) under Vertisols. Int.J.Curr.Microbiol.App.Sci. 10(01): 866-878. doi: https://doi.org/10.20546/ijcmas.2021.1001.105 